Cahiers de la recherche sur les droits

fondamentaux

$10 \mid 2012$

Esclavage et travail forcé

\title{
Traite des êtres humains et traite des migrants
}

Johanne Vernier

\section{OpenEdition}

Journals

Édition électronique

URL : https://journals.openedition.org/crdf/5256

DOI : $10.4000 /$ crdf. 5256

ISSN : 2264-1246

Éditeur

Presses universitaires de Caen

Édition imprimée

Date de publication : 1 novembre 2012

Pagination : 49-57

ISSN : 1634-8842

Référence électronique

Johanne Vernier, "Traite des êtres humains et traite des migrants », Cahiers de la recherche sur les droits fondamentaux [En ligne], 10 | 2012, mis en ligne le 01 décembre 2013, consulté le 14 novembre 2022. URL : http://journals.openedition.org/crdf/5256 ; DOI : https://doi.org/10.4000/crdf.5256 


\title{
Traite des êtres humains et traite des migrants
}

\author{
Johanne VERNIER \\ Doctorante en droit public à I'Université de Paris I
}

I. Une cible mal définie

A. Lutter contre la traite pour lutter contre l'exploitation

B. L'exploitation, une définition internationale incomplète

C. L'exploitation, une définition juridique absente du droit français

II. Des moyens employés pour atteindre une autre cible

A. Le renforcement de la lutte contre la prostitution

B. La priorité donnée à la lutte contre l'immigration irrégulière

Longtemps tolérée ou encadrée par la France, la traite des êtres humains est progressivement devenue, sous toutes ses formes, une pratique jugée criminelle.

Dans un premier temps, la France a renoncé en 1815, sous la pression de l'Angleterre, à la traite d'Africains aux fins d'esclavage à destination de ses colonies ${ }^{1}$; seront par la suite interdits le transport et le commerce d'esclaves au sein de ses colonies ou à destination de l'étranger. D'abord une simple contravention emportant confiscation du bâtiment et de sa cargaison ${ }^{2}$, la traite par voie maritime est finalement passible de travaux forcés, de réclusion ou d'emprisonnement en $1831^{3}$. Rarement sanctionnée en pratique, cette forme de traite disparaît néanmoins dans les années 1840. En 1848, l'esclavage est à son tour aboli $^{4}$. Au XXI ${ }^{\mathrm{e}}$ siècle, continue de peser sur la France l'obligation internationale de condamner tant l'esclavage que la traite à cette fin ${ }^{5}$.

Dans un deuxième temps, la Convention de 1910 a interdit le fait d'embaucher, entraîner ou détourner une femme majeure, contre son gré ${ }^{6}$, ou bien mineure aux fins de débauche ${ }^{7}$. Sauf cas d'esclavage sexuel, se trouvait ainsi qualifié de «traite» un comportement sans rapport avec la traite des esclaves. Cette interdiction a ensuite été étendue à la traite de tout enfant quel que soit son sexe $(1921)^{8}$, de toute femme majeure quel que soit son

1. Décret du 29 mars 1815 par lequel Napoléon abolit la «traite des noirs» et interdit d'introduire, pour être vendu dans les colonies françaises, "aucun noir provenant de la traite soit française, soit étrangère» (contravention).

2. Outre la confiscation du bâtiment et de sa cargaison (décret du 29 mars 1815) ainsi que l'interdiction pour le capitaine de reprendre tout commandement, l'ordonnance royale du 8 janvier 1817, puis la loi du 15 avril 1818, prévoyaient l'emploi des esclaves trouvés à bord à des «travaux d'utilité publique». À partir de 1831, l'État devait en principe les libérer et ne pouvait plus les engager que durant 7 ans maximum.

3. À titre de comparaison, en 1824, l'Angleterre considérait la traite comme un acte de piraterie passible de la peine capitale.

4. Décret du 27 avril 1848 .

5. Voir en particulier la Convention du 25 septembre 1926 relative à l'esclavage et la Convention supplémentaire du 30 avril 1956 relative à l'abolition de l'esclavage, de la traite des esclaves et des institutions et pratiques analogues à l'esclavage qui interdisent «tout acte de capture, d'acquisition ou de cession d'une personne en vue de la réduire en esclavage; tout acte d'acquisition d'un esclave en vue de le vendre ou de l'échanger; tout acte de cession par vente ou échange d'une personne acquise en vue d'être vendue ou échangée; ainsi qu'en général tout acte de commerce ou de transport d'esclaves, quel que soit le moyen de transport employé» (art. 7c).

6. Sont visés la fraude, les violences, les menaces, l'abus d'autorité ou tout autre moyen de contrainte.

7. Convention du 4 mai 1910 relative à la répression de la traite des blanches.

8. Convention du 30 septembre 1921 pour la suppression de la traite des femmes et des enfants. 
consentement $(1933)^{9}$ puis de toute personne, homme ou femme, quels que soient son âge ou son consentement $(1949)^{10}$. La France a retenu cette dernière approche, la plus large, contrairement à d'autres États estimant que le fait, pour une personne majeure, de consentir à se prostituer et d'être embauchée ou transportée à cette fin ne peut être qualifié de traite ${ }^{11}$. Le Code pénal punit encore aujourd'hui de 7 ans d'emprisonnement et 150 ooo euros d'amende le fait d'embaucher, entraîner ou détourner une personne en vue de la prostitution (art. 225-5, $3 \mathrm{du}$ Code pénal).

Dans un troisième et dernier temps, le Protocole dit de Palerme a interdit, en 2000, la traite aux fins non seulement d'esclavage ou d'exploitation de la prostitution mais aussi de travail forcé, de servitude, de toute forme d'exploitation sexuelle ou de prélèvement d'un organe $^{12}$. En conséquence, le Code pénal a incriminé en 2003 le fait de recruter, transporter, transférer, héberger ou accueillir une personne, afin d'en tirer un avantage quelconque, en sachant qu'elle est destinée soit à subir des faits de proxénétisme, d'agression ou d'atteinte sexuelles, d'exploitation de la mendicité ou de conditions indignes de travail ou d'hébergement, soit à être contrainte à commettre tout crime ou délit (art. 225-4-1 du Code pénal). Le traitant s'expose à 7 ans d'emprisonnement et 150 ooo euros d'amende ${ }^{13}$; s'il recourt aux menaces, à la force ou à la tromperie ou abuse de son autorité ou d'une situation de vulnérabilité, la peine encourue est plus élevée.

Malgré les efforts de la France pour condamner la traite sous toutes ses formes, la Commission nationale consultative des Droits de l'homme (CNCDH) constatait, en 2009, l'échec du dispositif mis en place ${ }^{14}$. Non seulement les victimes de traite sont rarement identifiées comme telles mais elles tendent en outre à être sanctionnées. La traite étant clairement et précisément incriminée, comment expliquer qu'il soit si difficile d'identifier les personnes qui en sont victimes? Pour quelles raisons nombre d'entre elles se trouvent sanctionnées au lieu d'être protégées?

\section{Une cible mal définie}

Un premier obstacle à une lutte efficace contre le phénomène criminel couramment désigné sous l'expression «traite des êtres humains» réside dans le fait qu'il n'est ni clairement ni précisément défini. L'identification et la protection des victimes s'en trouvent compromises ${ }^{15}$.

\section{A. Lutter contre la traite pour lutter contre l'exploitation}

Selon le droit français, la traite consiste à faciliter l'exploitation d'une personne; elle n'implique pas nécessairement que cette personne soit effectivement exploitée. Au contraire, en incriminant les actes facilitant l'exploitation d'une personne, l'infraction de traite vise à prévenir la commission de faits d'exploitation à son encontre. En d'autres termes, l'infraction de traite est une infraction-obstacle. Selon l'article 225-4-1 du Code pénal, le caractère criminel de la traite ne réside d'ailleurs pas dans le fait ordinaire de recruter, transporter, transférer, héberger ou accueillir une personne, même afin d'en tirer un avantage quelconque; il est emprunté au caractère lui-même criminel du projet poursuivi par le traitant, à savoir l'exploitation de la personne ainsi recrutée, transportée, etc.

En pratique, il s'avère toutefois difficile de constater des faits de traite, et a fortiori leur tentative ou préparation ${ }^{16}$, avant que la personne concernée ne commence au moins à être exploitée, révélant alors avec certitude le projet criminel du traitant. L'infraction de traite est alors vouée à s'appliquer en même temps que les infractions sanctionnant l'exploitation ou sa tentative (concours réel d'infractions). Dans cette hypothèse, il est cependant rare que les autorités répressives retiennent la qualification de traite, encore mal connue et relativement complexe. Ainsi, en 2006, deux condamnations seulement étaient prononcées sur le fondement de l'article 225-4-1; en 2007, trente-trois; et, en 2008 , dix-neuf ${ }^{17}$.

9. Convention du 11 octobre 1933 relative à la répression de la traite des femmes majeures.

10. Convention du 2 décembre 1949 pour la répression de la traite des êtres humains et de l'exploitation de la prostitution d'autrui.

11. Au sein de l'Union européenne, 11 États membres sur 27 n'ont pas ratifié la Convention de 1949.

12. Protocole du 15 novembre 2000, additionnel à la Convention contre la criminalité transnationale organisée, visant à prévenir, réprimer et punir la traite des personnes, en particulier des femmes et des enfants (dit Protocole de Palerme). La définition de la traite que consacre ce Protocole a été reprise par la Convention européenne du 16 mai 2005 sur la lutte contre la traite des êtres humains (dite Convention de Varsovie) et complétée par la Directive européenne 2011/36/UE du 5 avril 2011 concernant la prévention de la traite des êtres humains et la lutte contre ce phénomène ainsi que la protection des victimes et remplaçant la Décision-cadre 2002/629/JAI.

13. Loi n 2003-239 du 18 mars 2003 pour la sécurité intérieure, modifiée par la loi nº 2007-1631 du 20 novembre 2007 relative à la maîtrise de l'immigration, à l'intégration et à l'asile.

14. CNCDH, Avis sur la traite et l'exploitation des êtres humains en France, adopté le 18 décembre 2009 et présenté dans J. Vernier, La traite et l'exploitation des êtres humains en France, Paris, La documentation française (Les études de la CNCDH), 2010. L'avis comme l'étude qui l'accompagne sont disponibles à l'adresse suivante: www.cncdh.fr.

15. Voir J. Vernier, La traite et l'exploitation..., chap. 4.

16. Est incriminée l'association de malfaiteurs en vue de permettre la préparation des délits de traite (art. 450-1 sq. du Code pénal).

17. Voir J. Vernier, La traite et l'exploitation..., p. 111 (données provisoires). 
Étant admis que la répression de la traite ne se substitue pas à celle de l'exploitation mais la complète et que l'exploitation se trouve au cœur du phénomène criminel à combattre, il paraît essentiel d'incriminer et réprimer aussi et surtout l'exploitation. Or, si le droit français en vigueur présente une infraction générale de traite depuis 2003, il en va différemment de l'exploitation. Seules certaines de ses manifestations sont spécifiquement saisies par le droit pénal, telles que l'exploitation de la prostitution, de la mendicité ou de la vente à la sauvette ${ }^{18}$. Tous autres cas d'exploitation sont donc sanctionnés sur le fondement d'infractions-relais n'ayant pas vocation à les réprimer et n'en saisissant que certains aspects (conditions indignes de travail ou d'hébergement, travail dissimulé, violences, agression ou atteinte sexuelles, menaces, enlèvement, séquestration, extorsion de fonds, abus de faiblesse, aide au séjour irrégulier, etc. ${ }^{19}$ ).

\section{B. L'exploitation, une définition internationale incomplète}

Par exploitation, le Protocole de Palerme entend, «au minimum, l'exploitation de la prostitution d'autrui ou d'autres formes d'exploitation sexuelle, le travail et les services forcés, l'esclavage ou les pratiques analogues à l'esclavage, la servitude ou le prélèvement d'organes ${ }^{20}$. D'autres textes internationaux s'y rapportent plus spécifiquement. Ainsi, sont par ailleurs interdits et définis le travail ou les services forcés, l'esclavage et les institutions ou pratiques analogues à l'esclavage (servitude pour dettes, servage, etc.), qu'ils aient ou non un caractère sexuel ${ }^{21}$. La Cour européenne des Droits de l'homme (CEDH) a pour sa part précisé le sens à donner à la notion de servitude ${ }^{22}$. L'exploitation sexuelle, en particulier l'exploitation de la prostitution $^{23}$, et le prélèvement (illicite) d'organes ${ }^{24}$, bien qu'interdits, restent quant à eux à définir. Le droit international ne fournit de ce fait aucune définition générale de l'exploitation qui serait commune à l'ensemble des États, ce qui n'est pas sans nuire à leur coopération.

Parmi les formes d'exploitation les plus graves définies par le droit international, se trouvent le travail ou les services forcés, la servitude et l'esclavage. Selon la Cour européenne des Droits de l'homme, chacune correspond à un degré différent de gravité ${ }^{25}$. Un travail ou un service est «forcé» quand il est exigé d'une personne «sous la menace d'une peine quelconque» et pour lequel elle ne s'est pas offerte « de plein gré ${ }^{26}$; la servitude consiste en un travail forcé accompagné d'une négation particulièrement grave de la liberté de la personne ${ }^{27}$; et l'esclavage est «l'état ou la condition d'un individu sur lequel s'exercent les attributs du droit de propriété ou certains d'entre eux», plaçant l'esclave, privé de son autonomie, dans l'impossibilité de refuser de travailler ${ }^{28}$. Ces trois formes d'exploitation ont pour point commun le travail forcé, dessinant ainsi un continuum d'exploitation.

De nombreuses incertitudes demeurent toutefois ${ }^{29}$. Par exemple, le travail, même entendu largement et forcé, n'est pas commun à toute forme d'exploitation. Ainsi, l'esclavage n'emporte pas nécessairement la fourniture d'un travail ni d'un service. Il convient donc de chercher ailleurs les éléments invariants de l'exploitation, par exemple dans l'atteinte portée à la liberté de la personne, en particulier la liberté de son consentement, pour obtenir d'elle ce qu'elle n'aurait autrement pas accordé. De nouvelles difficultés sont alors rencontrées, notamment pour déterminer le degré de gravité que doit présenter une atteinte à la liberté pour emporter la qualification d'exploitation au sens pénal du terme; dans quelle mesure faut-il tenir compte de la situation de vulnérabilité ou de dépendance d'une personne; ou encore à partir de quels critères définir les notions de vulnérabilité et de dépendance. Mise à part une atteinte à la liberté, d'autres éléments sont également susceptibles de caractériser l'exploitation, comme la recherche d'un avantage par celui qui exploite une personne ou la gravité du préjudice subi par celle-ci ${ }^{30}$.

18. Art. 225-5 sq., 225-12-5 et 225-12-8 du Code pénal.

19. La délinquance forcée présente cette particularité que son auteur est généralement appréhendé en tant que complice de l'infraction commise, sous la contrainte, par la victime. Voir J. Vernier, La traite et l'exploitation..., p. 82-84.

20. Art. 3 a du Protocole de Palerme, 2000.

21. Voir en particulier la Convention du 25 septembre 1926 relative à l'esclavage, la Convention no 29 du 28 juin 1930 sur le travail forcé et la Convention supplémentaire du 30 avril 1956 relative à l'abolition de l'esclavage, de la traite des esclaves et des institutions et pratiques analogues à l'esclavage.

22. CEDH, Siliadin c. France, 26 juillet 2005, requête nº 73316/01, CEDH 2005-VII, § 123-128.

23. Voir J. Vernier, La traite et l'exploitation..., p. 42-44. Les textes interdisant l'exploitation sexuelle des enfants se font plus précis, comme la Convention du 20 novembre 1989 relative aux droits de l'enfant (art. 34), le Protocole du 25 mai 20oo, additionnel à la Convention relative aux droits de l'enfant, concernant la vente d'enfants, la prostitution des enfants et la pornographie mettant en scène des enfants (art. $2 b$ ), la Décision-cadre européenne 2004/68/JAI du 22 décembre 2003 (art. 2) et la Convention du 25 octobre 2007 sur la protection des enfants contre l'exploitation et les abus sexuels (art. 19-1c).

24. Voir le Protocole du 24 janvier 2002, additionnel à la Convention du Conseil de l'Europe sur les Droits de l'homme et la biomédecine, relatif à la transplantation d'organes et de tissus d'origine humaine (art. 13). Au $1^{\text {er }}$ janvier 2012, il n'était cependant pas ratifié par la France.

25. Voir CEDH, Siliadin c. France, $\$ 121,122,124$ et 126.

26. Convention $\mathrm{n}^{\circ} 29$ du 28 juin 1930 sur le travail forcé.

27. CEDH, Siliadin c. France, $\$ 123-124$.

28. Convention du 25 septembre 1926 relative à l'esclavage.

29. Voir J. Vernier, La traite et l'exploitation..., p. 45-53.

30. S'agissant des enfants, sont interdites toute forme d'exploitation économique ou sexuelle ainsi que toute autre forme d'exploitation préjudiciable à leur santé, leur sécurité ou leur moralité. Voir la Convention du 20 novembre 1989 relative aux droits de l'enfant (art. 32, 34 et 36); Convention $\mathrm{n}^{\circ} 182$ du 17 juin 1999 concernant l'interdiction des pires formes de travail des enfants et l'action immédiate en vue de leur élimination (art. 3 ). 


\section{L'exploitation, une définition juridique absente du droit français}

Le droit français ne définit pas davantage la notion d'exploitation que le droit international. En l'absence de dispositions pénales qui y soient spécifiques, sa répression varie selon l'infraction-relais appliquée ${ }^{31}$. Le Code pénal ne connaît pas même des infractions de travail forcé, de servitude ou d'esclavage, plaçant ainsi la France dans l'impossibilité de remplir ses obligations internationales s'agissant d'offrir aux personnes qui en sont victimes la reconnaissance et la protection auxquelles elles ont droit $^{32}$.

Tout d'abord, bien que réduire autrui en esclavage constitue un crime contre l'humanité sévèrement puni quand est visé un groupe de population civile dans le cadre d'une attaque généralisée ou systématique en exécution d'un plan concerté (art. 212-1 du Code pénal) ${ }^{33}$, aucune disposition n'incrimine l'esclavage en dehors de cette hypothèse. Par conséquent, pour que l'exercice d'un droit de propriété ou de certains de ses attributs sur une personne soit punissable - lorsqu'il échappe à la définition du crime contre l'humanité -, il faut que l'auteur adopte en outre un comportement incriminé par le droit pénal (violences, séquestrations, conditions indignes de travail ou d'hébergement, etc.). Ces infractions-relais ne permettent cependant pas de saisir l'esclavage dans tous ses éléments ni toute sa gravité ${ }^{34}$.

Dans ce contexte, la justice s'est trouvée démunie en 2007 pour signifier à ceux qui avaient acheté des nouveaunés à leurs mères - exerçant ainsi un droit de propriété sur eux -, pour ensuite les remettre à des parents adoptifs moyennant contrepartie, qu'ils avaient commis un acte d'une extrême gravité ${ }^{35}$. Afin d'y pallier, le juge pénal s'est efforcé de qualifier les faits non seulement de provocation à l'abandon de nouveau-nés et d'atteinte à leur état civil mais encore de traite ${ }^{36}$. Pour ce faire, il a été estimé que les auteurs avaient recruté, transporté, transféré, hébergé ou accueilli leurs mères biologiques, pour en tirer profit, en vue de les contraindre à abandonner leur enfant ou dissimuler leur filiation (traite aux fins de délinquance forcée). La qualification de traite aurait toutefois dû être écartée, la preuve d'une contrainte exercée sur elles pour abandonner leur enfant ou dissimuler leur filiation n'ayant pas été rapportée ${ }^{37}$. Cet exemple confirme que le droit en vigueur est voué à laisser impunis l'esclavage comme la traite à cette fin.

Ensuite, la France a été condamnée par la Cour européenne des Droits de l'homme en 2005, au motif que le droit pénal en vigueur avant 2003 ne permettait de lutter de manière effective ni contre le travail forcé ni contre la servitude. En l'espèce, une jeune étrangère était arrivée en France alors qu'elle était mineure et avait dû effectuer de multiples tâches domestiques, des années durant, sans compter ses heures, sans repos, ni congés, ni rémunération, alors qu'elle était isolée de sa famille, se trouvait en situation irrégulière et craignait une arrestation (une crainte d'ailleurs entretenue par les auteurs) ${ }^{38}$. À l'issue de la procédure pénale engagée en France, les auteurs avaient été condamnés pour non-rétribution de son travail en ayant abusé de sa situation de vulnérabilité ou de son état de dépendance (art. 225-13 du Code pénal) mais relaxés s'agissant du caractère indigne de ses conditions de travail et d'hébergement (art. 225-14 du Code pénal).

Depuis 2003 , la preuve de ces délits a été facilitée ${ }^{39}$, la peine encourue a été aggravée ${ }^{40}$ et la jurisprudence a posé le principe selon lequel les conditions d'exercice d'un travail forcé sont nécessairement indignes ${ }^{41}$. Les dispositions prévues aux articles 225-13 et 225-14 demeurent cependant insatisfaisantes s'agissant de réprimer tant le travail forcé que la servitude ${ }^{42}$. Le recours à la contrainte ou les atteintes portées aux libertés de la personne n'en sont ni un élément constitutif ni une circonstance aggravante, la notion de dignité persiste à être étroitement entendue par certains juges, les peines prononcées sur ce fondement ne dépassent généralement pas quelques mois d'emprisonnement avec sursis ${ }^{43}$, les victimes disposent de 3 ans seulement pour déposer plainte, etc. Ces infractions-relais

31. Voir CNCDH, Avis sur la traite..., recommandations no 9 à 11; voir J. Vernier, La traite et l'exploitation..., p. 97-107.

32. Rappelons que l'esclavage et la servitude font l'objet d'une interdiction internationale absolue, ne supportant aucune dérogation (art. 4 et 15-2 de la Convention européenne de sauvegarde des Droits de l'homme et des libertés fondamentales).

33. Depuis l'entrée en vigueur de la loi nº 2010-930 du 9 août 2010 portant adaptation du droit pénal à l'institution de la Cour pénale internationale, il n'est plus nécessaire de rapporter la preuve que la réduction en esclavage a été inspirée par des motifs politiques, philosophiques, sociaux ou religieux.

34. Depuis 2005, plusieurs propositions de loi ont été déposées au Sénat et à l'Assemblée nationale afin d'incriminer spécifiquement l'esclavage.

35. TGI Bobigny, $13^{\mathrm{e}}$ chambre, 2 février 2007 ( $\mathrm{n}^{\circ} 0419121163$ ).

36. Art. 225-4-1, 227-12, 227-13, al. 1 du Code pénal.

37. Voir J. Vernier, La traite et l'exploitation..., p. 72-73.

38. CEDH, Siliadin c. France.

39. La preuve de l'«abus» d'une situation de vulnérabilité ou d'un état de dépendance n'a plus à être rapportée. Il suffit de prouver que l'auteur avait connaissance ou ne pouvait ignorer cette situation ou cet état. De plus, un migrant à son arrivée en France ou un mineur sont légalement présumés se trouver dans cette situation ou cet état.

40. Elle est passée de 2 à 5 ans d'emprisonnement et de 75000 à 150000 euros d'amende.

41. Cass. crim., 13 janvier 2009, $\mathrm{n}^{\circ}$ 08-80787.

42. En ce sens, voir H. Clément, intervention présentée dans le cadre de l'atelier «Répression: définition et incrimination pénale» lors de la réunion technique BIT-CNCDH des 26-27 octobre 2009, Traite des êtres humains et travail forcé en France. Se pencher sur les formes d'exploitation autres que sexuelles, dont les actes ont été publiés en ligne le 13 avril 2012, http://www.ilo.org/sapfl/Informationresources/ILOPublications/ WCMS_177938/lang--fr/index.htm, p. 90.

43. Voir J. Vernier, La traite et l'exploitation..., p. 76-82 
ne comblent aucunement le vide juridique laissé par les infractions manquantes de travail forcé et de servitude.

Enfin, les dispositions visant a priori spécifiquement l'exploitation de la prostitution, de la mendicité et de la vente à la sauvette ne sanctionnent pas uniquement des faits de travail forcé ou de servitude. Les infractions relatives au proxénétisme et à l'«exploitation» de la mendicité ou de la vente à la sauvette poursuivent deux objectifs à la fois ${ }^{44}$. Dans leur forme simple, elles permettent de faire obstacle à l'exercice de la prostitution et de la mendicité, considérées comme des comportements déviants, et à la vente à la sauvette, incriminée en $2011^{45}$. Sous leur forme aggravée, ces infractions peuvent caractériser une situation d'exploitation dans certaines circonstances, en particulier lorsque l'auteur use de la force, de menaces ou de la tromperie ou abuse de son autorité ou d'une situation de vulnérabilité.

Si la répression de ces infractions, dans leur forme simple, présente l'avantage de pouvoir sanctionner des situations d'exploitation sans avoir à rapporter la preuve de l'ensemble de ses éléments constitutifs, cela n'est pas sans inconvénients pour les victimes. Outre le fait qu'elles apparaissent elles-mêmes comme les auteurs de comportements considérés comme déviants (prostitution, mendicité) ou délictuels (vente à la sauvette), elles peuvent également être appréhendées comme les auteurs de proxénétisme, d'« exploitation» de la mendicité ou d' « exploitation » de la vente à la sauvette. Par exemple, une victime d'exploitation de la prostitution peut être qualifiée à la fois de victime et d'auteur de faits de proxénétisme au motif qu'elle a aidé, d'une manière quelconque, autrui à se prostituer ${ }^{46}$. S'il lui est possible d'échapper à une sanction en prouvant qu'elle a été contrainte à fournir cette aide sans avoir pu y résister (art. 122-2 du Code pénal), il est difficile en pratique d'en convaincre le juge, en particulier lorsque la contrainte exercée sur elle a été d'ordre psychologique ${ }^{47}$.

L'infraction de traite n'a pas pour fonction de se substituer à la répression de l'exploitation mais de la compléter. Or, l'exploitation n'est elle-même que très partiellement incriminée; aucune infraction n’en sanctionne spécifiquement les formes les plus graves telles que le travail forcé, la servitude et l'esclavage. Les personnes qui en sont victimes peinent de ce fait à s'identifier comme telles ou à l'être par les services de détection et de répression. Et la protection offerte aux victimes d'exploitation varie selon l'infraction-relais appliquée plutôt que la gravité des faits subis. Ni lisible ni prévisible, la loi pénale doit être clarifiée et complétée, comme le recommande la $\mathrm{CNCDH}^{48}$.

\section{Des moyens employés pour atteindre une autre cible}

Outre la difficulté rencontrée pour identifier les victimes d'exploitation et de traite en l'absence d'une définition claire et précise du phénomène criminel concerné, il est un autre obstacle majeur à leur protection: nombre de victimes sont elles-mêmes appréhendées comme des délinquantes. La lutte contre l'exploitation et la traite, impliquant de protéger les personnes qui en sont victimes, semble s'effacer devant la lutte contre les infractions susceptibles d'être commises par elles (racolage public, entrée ou séjour irréguliers, vente à la sauvette, vol, etc.). En observant, par exemple, les interactions entre, d'une part, la politique criminelle relative à la traite et, d'autre part, les politiques criminelles relatives à la prostitution et à l'immigration irrégulière, qui poursuivent des objectifs a priori contradictoires - protéger et sanctionner -, il apparaît en fait que les mesures adoptées ou mises en œuvre dans le cadre de la lutte contre l'exploitation et la traite partagent, dans une certaine mesure, l'objectif de sanctionner les personnes qui en sont victimes, voire renforcent les moyens de les sanctionner.

\section{A. Le renforcement de la lutte contre la prostitution}

En 2003, tandis que la Loi pour la sécurité intérieure introduisait dans le Code pénal une nouvelle infraction générale de traite, la lutte contre la prostitution était par la même occasion renforcée, principalement, par deux mesures ${ }^{49}$

Tout d'abord, la contravention de racolage public ${ }^{50}$ est devenue un délit passible de 2 mois d'emprisonnement et 3750 euros d'amende. Est visée toute personne qui, «par tout moyen, y compris par une attitude passive, [procède] publiquement au racolage d'autrui en vue de l'inciter à des relations sexuelles en échange d'une rémunération ou d'une promesse de rémunération » (art. 225-10-1 du Code pénal). Alors que seul le racolage actif était auparavant interdit, le racolage passif expose désormais son auteur à une peine identique, y compris en cas de simple tentative. En 1994, la répression du racolage passif avait pourtant été abandonnée «en raison de l'imprécision de l'élément constitutif de cette infraction qui aboutissait à une application aléatoire par les services de constatation ${ }^{51}$.

44. Art. 225-5 sq., 225-12-5 sq., 225-12-8 sq. du Code pénal.

45. Loi no 2011-267 du 14 mars 2011 d'orientation et de programmation pour la performance de la sécurité intérieure.

46. Le 21 octobre 2003 , le tribunal de grande instance de Paris ( $16^{\mathrm{e}}$ chambre) a par exemple condamné une jeune femme à 2 ans d'emprisonnement avec sursis pour proxénétisme aggravé au motif qu'elle avait pris en charge plusieurs femmes se prostituant et recueilli leurs gains avant de les envoyer par mandat pour le compte de celui qui les exploitait. Elle avait elle-même été achetée, privée de son passeport et contrainte, sous la menace de représailles sur son fils, non seulement à se prostituer mais aussi à accomplir ces autres tâches qui lui étaient reprochées.

47. Voir J. Vernier, La traite et l'exploitation..., p. 210-211.

48. Ibid., chap. 2

49. Loi $\mathrm{n}^{\circ} 2003-239$ du 18 mars 2003 pour la sécurité intérieure.

50. Ancien article R. 625-8 du Code pénal.

51. Réponse du garde des Sceaux à la question écrite no 19596, JOAN, 9 janvier 1995, p. 212. 
Ce revirement a été justifié par la nécessité non seulement de sauvegarder la tranquillité et la moralité publiques mais aussi de lutter plus efficacement contre la criminalité transnationale organisée, que la visibilité accrue de personnes prostituées et étrangères semblait révéler. Sanctionner les prostitués sur le fondement du racolage public devait priver le proxénétisme de sa source de profit et ainsi faire échec à la traite ${ }^{52}$.

En pratique, les prostitués les plus visibles, y compris ceux d'entre eux victimes d'exploitation de la prostitution ou de traite, ont effectivement été et continuent d'être arrêtés et placés en garde à vue sur le fondement de l'article 22510-1. En 2005, 996 personnes étaient condamnées pour racolage public ${ }^{53}$. Par la suite, le parquet a privilégié le rappel à la loi ou le classement sans suite, à condition de ne pas récidiver avant un certain délai ou de s'orienter vers une ou plusieurs structures sanitaires, sociales ou professionnelles en vue d'un « reclassement ${ }^{54}$. En 2008, trois fois moins de condamnations étaient par conséquent prononcées ${ }^{55}$.

La répression subie par les prostitués s'avère toutefois plus policière que judiciaire, voire "administrative» lorsqu'ils sont étrangers et en situation irrégulière. La Commission Citoyens-Justice-Police ${ }^{56}$ concluait, en 2006, à l'existence d'une " justice policière», «en marge des principes de l'État de droit», constatant que «les preuves [du racolage public] sont appréciées par la seule police, la garde à vue joue le rôle d'une courte peine, la confiscation de l'argent tient lieu d'amende, le rappel à la loi de jugement, les conditions dont il est assorti de mise à l'épreuve, le STIC de casier judiciaire ${ }^{57}$. En cas de contrôle fondé sur le racolage public, les étrangers en situation irrégulière peuvent en outre être placés en rétention administrative en vue de leur éloignement du territoire ${ }^{58}$. Des prostitués passent ainsi plusieurs mois par an en détention ou rétention.

Outre le fait que le délit de racolage public mette à mal les principes de légalité, de présomption d'innocence, du contradictoire et de proportionnalité - en cela, le régime actuel de la prostitution visible n'est pas moins arbitraire que ceux qui l'ont précédé ${ }^{59}$-, les arguments abondent pour exiger que soit abandonnée la stratégie qui consiste à sanctionner ceux qui devraient être protégés. Pèse sur la France l'obligation internationale de protéger, de manière effective, toute personne contre l'exploitation et la traite. Appréhendées comme des délinquantes, les victimes d'exploitation de la prostitution et de traite tendent à éviter les services de détection et de répression au lieu de rechercher leur protection. Car repoussés dans des espaces publics isolés et, surtout, dans l'illégalité, l'ensemble des prostitués se trouvent davantage exposés à la violation de leurs droits, y compris à l'exploitation et la traite. C'est pourquoi, en 2009, la CNCDH recommandait vivement d'abroger le délit de racolage public ${ }^{60}$.

Ensuite, la Loi pour la sécurité intérieure a également interdit de solliciter, accepter ou obtenir, en échange d'une rémunération ou d'une promesse de rémunération, des relations sexuelles de la part d'une personne majeure qui se livre à la prostitution alors qu'elle " présente une particulière vulnérabilité, apparente ou connue de son auteur, due à une maladie, à une infirmité, à une déficience physique ou psychique ou à un état de grossesse» (art. 225-12-1 du Code pénal). Auparavant, seul le recours à la prostitution d'un mineur entraînait 3 ans d'emprisonnement et 45000 euros d'amende ${ }^{61}$.

Contrairement au délit de racolage public, le délit de recours à la prostitution de mineurs ou de majeurs en situation de particulière vulnérabilité est très rarement appliqué. En 2008, seulement vingt-six condamnations étaient prononcées ${ }^{62}$. Malgré l'ineffectivité flagrante de cette interdiction, il a récemment été proposé de l'étendre à tout recours à la prostitution d'autrui au motif de lutter plus efficacement contre l'exploitation et la traite ${ }^{63}$. Si cette proposition de loi devait être adoptée, il est fort probable que les clients concernés ne seraient pas davantage sanctionnés qu'ils ne le sont déjà quand ils s'adressent aux prostitués parmi les plus vulnérables. Surtout, en prohibant toute prostitution, l'infraction proposée aurait pour conséquence d'aggraver les effets déjà produits par le délit de racolage public et, de ce fait, favoriserait - au lieu de prévenir - l'exploitation et la traite.

52. Circulaire du 3 juin 2003 sur l'application de la loi n² 2003-239 pour la sécurité intérieure (NOR/JUS/D/03/30082/C).

53. Voir J. Vernier, La traite et l'exploitation..., p. 216.

54. Art. 40-1 et 41-1 du Code de procédure pénale.

55. Voir J. Vernier, La traite et l'exploitation..., p. 216 (données provisoires).

56. La Commission regroupait alors la Ligue des Droits de l'homme, le Mouvement contre le racisme et pour l'amitié entre les peuples, le Syndicat des avocats de France et le Syndicat de la magistrature.

57. Commission Citoyens-Justice-Police, De nouvelles zones de non droit. Des prostituées face à l'arbitraire policier, $2006, \mathrm{p} .18$.

58. Selon la circulaire du 3 juin 2003 présentant le délit de racolage public diffusée par le ministère de la Justice, «il n'y aurait que des avantages à ce que, pendant la durée de l'enquête, la personne fasse l'objet d'une procédure administrative destinée à permettre sa reconduite à la frontière, ce qui évitera ainsi des poursuites pénales» $(\$ 2$ 2.3.2).

59. Voir J. Vernier, "La prostitution des femmes saisie par la norme pénale», in Figures de femmes criminelles. De l'Antiquité à nos jours, L. Cadiet, F. Chauvaud et al. (dir.), Paris, Publications de la Sorbonne, 2010, p. 223-234.

60. $\mathrm{CNCDH}$, Avis sur la traite..., recommandation $\mathrm{n}^{\circ} 43$.

61. Loi no 2002-305 du 4 mars 2002 relative à l'autorité parentale. Avant 2002, seul le recours à la prostitution d'un mineur âgé de moins de 15 ans était sanctionné en tant qu'atteinte sexuelle sur mineur, avec cette circonstance aggravante qu'une rémunération avait été versée.

62. Voir J. Vernier, La traite et l'exploitation..., p. 111 (données provisoires).

63. Proposition de loi $\mathrm{n}^{\circ} 4057$ du 7 décembre 2011 visant à responsabiliser les clients de la prostitution et à renforcer la protection des victimes de la traite des êtres humains et du proxénétisme. 
Plutôt que d'interdire la prostitution en sanctionnant tout client, il serait sans doute préférable, d'une part, de garantir la répression de tout abus subi par les prostitués et déjà condamné par le droit pénal, en particulier les agressions ou atteintes sexuelles ${ }^{64}$, que l'auteur ait versé ou non une rémunération. D'autre part, il conviendrait de limiter la sanction pénale des clients, en tant que tels, aux cas de recel d'exploitation. En dehors de cette hypothèse, le droit international encourage d'ailleurs à adopter des mesures «d'ordre éducatif, social ou culturel » visant à décourager la demande en ce qu'elle favorise l'exploitation $^{65}$. Il s'agirait donc de sanctionner uniquement les personnes, physiques ou morales, qui bénéficient, «en connaissance de cause ", de l'activité exercée ou du service fourni par une victime d'exploitation, une fois l'exploitation clairement et précisément définie ${ }^{66}$.

En renforçant la répression de la prostitution, non seulement des victimes d'exploitation de la prostitution ou de traite à cette fin se sont trouvées sanctionnées mais la criminalisation et la stigmatisation accrue de la prostitution ont également placé l'ensemble des prostitués dans une situation de vulnérabilité accrue, favorisant la commission d'abus à leur égard. C'est pourquoi la CNCDH appelle à repenser, en profondeur, le régime actuel de la prostitution, en privilégiant notamment l'application du droit commun aux prostitués qu'ils soient auteurs (exhibitionnisme, troubles à l'ordre public, etc.) ou victimes d'infractions (exploitation, traite, violences, agressions ou atteintes sexuelles, vol, extorsions de fonds, abus de faiblesse, discrimination, etc. $)^{67}$.

\section{B. La priorité donnée à la lutte contre l'immigration irrégulière}

Au tournant du XXI siècle, moins préoccupés par la nécessité de lutter contre l'exploitation sur leur propre territoire que de lutter contre l'immigration de ceux qui en sont victimes, les États ont mis l'accent sur la traite - comprise comme une forme de criminalité transnationale - plutôt que l'exploitation. Dans ce contexte, ils ont été encouragés à renforcer le contrôle des frontières ${ }^{68}$ et la lutte contre l'immigration irrégulière ${ }^{69}$. Or, si le contrôle des frontières peut être une occasion d'identifier certaines des victimes de traite, à condition d'y former les services concernés, la lutte contre l'immigration irrégulière conduit à sanctionner et non protéger celles parmi elles se trouvant en situation irrégulière.

Tout d'abord, une certaine confusion entre traite et aide à la migration irrégulière est entretenue par les textes, de telle façon que nombre de cas de traite sont qualifiés d'aide à la migration irrégulière. Une telle confusion est loin d'être sans conséquence pour les migrants irréguliers victimes de traite.

En principe, traite et aide à la migration irrégulière sont deux comportements distincts. Depuis 2000, ils sont d'ailleurs saisis par différents textes à l'échelle internationale ${ }^{70}$ comme nationale. Ainsi, le droit français punit de 5 ans d'emprisonnement et 30 ooo euros d'amende le fait, par aide directe ou indirecte, de faciliter ou tenter de faciliter l'entrée, la circulation ou le séjour irréguliers d'un étranger en France ou dans un autre État, appartenant à l'espace Schengen ou partie au Protocole des Nations unies contre le trafic illicite de migrants (art. L. 622-1 du Code de l'entrée et du séjour des étrangers et du droit d'asile (CESEDA)).

Les infractions de traite et d'aide à la migration irrégulière se distinguent a priori tant par leurs éléments constitutifs que par l'intérêt qu'elles protègent. Toutes les victimes de traite ne sont pas des migrants irréguliers et, inversement, tout migrant irrégulier n'est pas victime de traite. En effet, la traite n'implique pas nécessairement le franchissement d'une frontière et les étrangers aidés à migrer de façon irrégulière ne le sont pas nécessairement aux fins d'exploitation. Quant à l'intérêt protégé par les infractions de traite et d'aide à la migration irrégulière, la première constitue une atteinte portée aux droits fondamentaux de la personne tandis que la seconde est considérée comme portant gravement préjudice, en premier lieu, à l'État ${ }^{71}$. Dans un cas, le migrant concerné est d'abord une victime d'infraction qui, dans la mesure du possible, ne doit pas être sanctionnée pour les infractions qu'il a éventuellement commises dans ce contexte ${ }^{72}$. Dans l'autre, il est avant toute autre chose appréhendé comme l'auteur d'une infraction à la législation relative à l'immigration ${ }^{73}$.

64. Parmi les atteintes sexuelles figure le recours à la prostitution d'un mineur. Pour une critique de l'article 225-12-1 du Code pénal dont le texte ne protège pas tout mineur contre la prostitution, voir J. Vernier, La traite et l'exploitation..., p. 74-75 et 303-304.

65. Art. 9-5 du Protocole de Palerme. Voir aussi l'art. 6 de la Convention de Varsovie et l'art. 18-1 de la Directive européenne 2011/36/UE.

66. Art. 19 de la Convention de Varsovie; art. 18-4 de la Directive européenne 2011/36/UE; CNCDH, Avis sur la traite..., recommandation no 8; J. Vernier, La traite et l'exploitation..., p. 94-97.

67. CNCDH, Avis sur la traite..., recommandations n $\mathrm{n}^{\circ} 78$ et 79; voir J. Vernier, La traite et l'exploitation..., p. 299-307.

68. Art. 11-1 du Protocole de Palerme; art. 7-1 de la Convention de Varsovie.

69. Voir par exemple la Décision-cadre 2002/946/JAI du 28 novembre 2002 visant à renforcer le cadre pénal pour la répression de l'aide à l'entrée, au transit et au séjour irréguliers (préambule, $\$ 2$ et 5 ).

70. Voir en particulier le Protocole du 12 décembre 2000, additionnel à la Convention contre la criminalité transnationale organisée, contre le trafic illicite de migrants par terre, mer et air; la Directive européenne 2002/90/CE du 28 novembre 2002 définissant l'aide à l'entrée, au transit et au séjour irréguliers; et la Décision-cadre 2002/946/JAI du même jour visant à renforcer le cadre pénal pour la répression de l'aide à l'entrée, au transit et au séjour irréguliers.

71. Préambule du Protocole contre le trafic illicite de migrants par terre, mer et air.

72. Voir en particulier la Convention de Varsovie (art. 26) et la Directive européenne 2011/36/UE d (art. 8).

73. Art. L. 511-1 et L. 621-1 du CESEDA. 
Toutefois, lorsqu'un migrant irrégulier est victime de traite, les définitions légales de la traite et de l'aide à la migration irrégulière se chevauchent partiellement. Ainsi, un passeur s'expose à une peine plus sévère lorsque l'aide à la migration irrégulière qu'il a fournie a eu «pour effet de soumettre les étrangers à des conditions de vie, de transport, de travail ou d'hébergement incompatibles avec la dignité de la personne humaine» (art. L. 622-5 du CESEDA) ${ }^{74}$. Un tel comportement entraîne une peine de 10 ans d'emprisonnement et 750 ooo euros d'amende, soit une peine similaire à celle encourue en cas de traite d'un migrant ${ }^{75}$. En pratique, alors que ces deux infractions protègent deux intérêts distincts et peuvent par conséquent être appliquées ensemble aux mêmes faits, le cas échéant, celle d'aide à la migration irrégulière tend à être appliquée au détriment de celle de traite ${ }^{76}$. Les migrants irréguliers victimes de traite tendent de ce fait à être perçus uniquement comme des délinquants.

Ensuite, le risque d'appréhender des faits de traite sur le fondement de la lutte contre l'immigration irrégulière est accru s'agissant des formes d'exploitation autres que sexuelles. Par exemple, alors que l'Office central pour la répression de la traite des êtres humains (OCRTEH) et l'Office central pour la répression des violences aux personnes (OCRVP) sont deux offices centraux dédiés à la lutte contre l'exploitation sexuelle, aucun office central n'a pour mandat de lutter contre les autres formes d'exploitation. Il arrive que l'Office central pour la répression de l'immigration illégale et de l'emploi des étrangers sans titre (OCRIEST) et l'Office central de lutte contre le travail illégal (OCLTI) aient à traiter ce type de dossier mais, le cas échéant, les faits sont plus volontiers qualifiés d'aide à la migration irrégulière ou de travail dissimulé, au mieux de conditions indignes de travail ou d'hébergement ${ }^{77}$. En ce cas, les étrangers en situation irrégulière qui en ont fait l'objet sont généralement éloignés du territoire.

Dans une certaine mesure, il en va de même pour les inspecteurs du travail qui, bien qu'ayant pour mission de protéger tous les travailleurs quelles que soient leur nationalité ou leur situation administrative, sont régulièrement appelés à participer à des opérations conjointes contre l'emploi des étrangers sans titre, qui aboutissent à l'éloignement des travailleurs concernés. En inscrivant la lutte contre le travail illégal dans le cadre de la lutte contre l'immigration irrégulière, priorité est donnée à l'éloignement des étrangers trouvés en situation irrégulière lors de ces opérations ${ }^{78}$. En 2009, la Commission d'experts de l'Organisation internationale du travail (OIT) a appelé la France à abandonner ce type d'opérations, jugées incompatibles avec les méthodes de contrôle et les objectifs de l'inspection du travail ${ }^{79}$. La CNCDH s'en est fait l'écho en $2009^{80}$.

Enfin, les dispositions prévues pour admettre au séjour les étrangers victimes ou témoins de faits de traite ou de proxénétisme reconnaissent moins un statut de victime aux étrangers concernés qu'il ne confirme le statut précaire des étrangers en situation irrégulière. En effet, l'article L. 316-1 du CESEDA, qui prévoit la délivrance d'une carte de séjour temporaire "vie privée et familiale » aux étrangers ayant déposé plainte ou témoigné contre les auteurs de faits de traite ou de proxénétisme, n'a jamais eu vocation à garantir la protection de l'ensemble des étrangers victimes d'exploitation ou de traite ${ }^{81}$. Il s'agit simplement d'un outil mis à disposition des autorités répressives pour recueillir les éléments utiles à la condamnation de la traite et du proxénétisme ${ }^{82}$. Sauf exception ${ }^{83}$, la menace d'un éloignement n'est que suspendue, jusqu'à l'issue de la procédure pénale engagée contre les auteurs. Ce faisant, l'article L. 316-1 se contente d'accorder une sorte de sursis administratif avec mise à l'épreuve à des étrangers victimes d'exploitation ou de traite mais appréhendés comme des délinquants.

Le droit international fait pourtant peser sur la France l'obligation internationale de protéger, de manière effective, toute victime d'exploitation ou de traite. Tout étranger devrait par conséquent non seulement recevoir une

74. Voir aussi l'art. 6-3 du Protocole contre le trafic illicite de migrants par terre, mer et air.

75. L'article 225-4-2, $4^{\circ}$ du Code pénal prévoit une peine de 10 ans d'emprisonnement et 1500 ooo euros d'amende lorsque les faits de traite incriminés à l'article 225-4-1 sont commis «à l'égard d'une personne qui se trouvait hors du territoire de la République ou lors de son arrivée sur le territoire de la République».

76. L'infraction d'aide à la migration irrégulière est mieux connue des autorités répressives et plus facile à prouver. Peu importe le comportement adopté par l'auteur (recrutement, transport, transfert, hébergement, accueil ou autre) dès lors qu'il a pour résultat d'aider un étranger à migrer de façon irrégulière. De plus, à défaut d'établir le caractère indigne de ses conditions de travail ou d'hébergement, celui de ses «conditions de vie» suffit.

77. Voir J. Vernier, La traite et l'exploitation..., p. 120-123.

78. Voir par exemple les circulaires des 26 mars et 24 décembre 2008 sur la lutte contre le travail illégal intéressant des ressortissants étrangers mise en œuvre d'opérations conjointes selon lesquelles: «les opérations de lutte contre le travail illégal intéressant les ressortissants étrangers participent directement de la lutte contre l'immigration irrégulière. À cet égard, il importe que toutes les dispositions soient prises à chacun des niveaux impliqués (services de sécurité intérieure, bureaux des étrangers des préfectures) pour faire en sorte que les interpellations des étrangers en situation irrégulière aboutissent à des éloignements effectifs».

79. BIT, Rapport de la Commission d'experts pour l'application des conventions et recommandations, 2009, p. 510-511.

80. CNCDH, Avis sur la traite..., recommandations n ${ }^{\circ}$ 12, 17, 80 et 81; voir J. Vernier, La traite et l'exploitation..., p. 123-127 et 307-309.

81. En 2009, seulement 56 cartes de séjour temporaire étaient délivrées sur le fondement de l’article L. 316-1 du CESEDA et 43 renouvelées (données provisoires). Voir J. Vernier, La traite et l'exploitation..., p. 145.

82. Selon l'article 8-1a de la Directive européenne 2004/81/CE du 29 avril 2004 relative au titre de séjour délivré aux ressortissants de pays tiers qui sont victimes de la traite des êtres humains, les États membres délivrent ou renouvellent le titre de séjour concerné après avoir examiné «s'il est opportun de prolonger son séjour sur [leur] territoire aux fins de l'enquête ou de la procédure judiciaire».

83. En cas de condamnation définitive des auteurs, une carte de résident peut être délivrée à l'étranger qui a déposé plainte ou témoigné contre eux (art. L. 316-1 du CESEDA) 
protection égale à celle accordée aux ressortissants français ${ }^{84}$ mais encore se voir reconnaître le droit de séjourner sur le territoire français dès lors que l'effectivité de ses droits en dépend ${ }^{85}$. Le statut de victime d'exploitation ou de traite devrait jouer un rôle décisif sur celui d'étranger, et non l'inverse. Or, à ce jour, la situation irrégulière des étrangers victimes d'exploitation ou de traite fait obstacle au bénéfice de certaines mesures, notamment celles censées garantir leur accès à la justice (comme l'aide juridictionnelle ou l'accès à la Commission d'indemnisation des victimes d'infraction ${ }^{86}$ ), et les expose à une mesure d'éloignement du territoire susceptible de priver de toute effectivité leurs droits d'accéder à la justice ou d'être rétablis dans leurs droits économiques et sociaux.

En primant sur la lutte contre l'exploitation et la traite, la lutte contre l'immigration irrégulière conduit à sanctionner des étrangers victimes de violations graves de leurs droits fondamentaux, au lieu de les protéger. C'est pourquoi la $\mathrm{CNCDH}$ appelle à dissocier migration irrégulière, exploitation et traite. De manière plus générale, elle recommande de repenser les politiques migratoires actuelles qui, en fermant les frontières et criminalisant la migration irrégulière, placent les migrants irréguliers dans une situation de vulnérabilité propice à la violation de leurs droits, y compris l'exploitation et la traite ${ }^{87}$.

Derrière l'expression «traite des êtres humains » apparaît ainsi une triste réalité. Le phénomène criminel qui devrait être en priorité combattu - l'exploitation d'une personne, en particulier le travail forcé, la servitude et l'esclavage - reste pour une large part indéfini et privé de réponse satisfaisante. Ceux qui en sont victimes peinent, en conséquence, à recevoir la protection à laquelle ils devraient avoir droit, quand ils ne sont pas sanctionnés en application de politiques criminelles paradoxalement renforcées ou mises en œuvre au nom d'une lutte efficace contre la traite. Ce faisant, le dispositif actuel n'échoue pas seulement à atteindre l'un des objectifs que le droit international lui assigne, la protection de toute personne contre l'exploitation et la traite. Il permet également qu'un objectif exactement contraire soit atteint, la sanction des personnes qui en sont victimes. Force est de constater qu'il ne s'agit pas d'un simple effet "pervers».

84. $\mathrm{CNCDH}$, Avis sur la traite..., recommandation $\mathrm{n}^{\circ} 25$.

85. Ibid., recommandations $\mathrm{n}^{\circ} 45$ et 66; voir J. Vernier, La traite et l'exploitation..., p. 218-229 et 271-277.

86. La Directive 2011/36/UE impose d'y remédier (art. 12-2 et 17). Voir aussi CNCDH, Avis sur la traite..., 2009, recommandations no 33 et 39 ; J. Vernier, La traite et l'exploitation..., p. 182-185 et 200-202. 